\title{
Extended Producer Responsibility in the Construction Sector through Blockchain, BIM and Smart Contract Technologies
}

\author{
Arghavan Akbarieh ${ }^{1}$, William Carbone ${ }^{2}$, Markus Schäfer ${ }^{1}$, Danièle Waldmann ${ }^{3}$, Felix Norman Teferle ${ }^{1}$ \\ ${ }^{1}$ Institute of Civil and Environmental Engineering, University of Luxembourg, Luxembourg, Luxembourg \\ Email: arghavan.akbarieh@uni.lu, markus.schaefer@uni.lu,norman.teferle@uni.lu \\ ${ }^{2}$ Global Automotive, Aerospace and Defence, IBM, Bratislava, Slovakia \\ Email: williamcarbone@sk.ibm.com \\ ${ }^{3}$ Institute of Civil and Environmental Engineering, University of Luxembourg, Esch-sur-Alzette, Luxembourg \\ Email: daniele.waldmann@uni.lu
}

\begin{abstract}
Despite the enormous amount of raw or secondary materials flowing within the construction industry, the actual available volume of materials and their respective End-ofLifecycle (EoL) treatment is not regulated nor uniform. On top of that, the EoL responsibility of different stakeholders after the future building deconstruction is confusing and disputable. Consequently, different sustainability policies and metrics suffer from inaccurately reported volumes of circulating materials in the economy. Hence, this article aims to find a new way to improve and regulate the EoL treatment of recyclable materials and to create value for them. The ultimate goal of the proposed framework is to make original manufacturers responsible for the EoL treatment of their recyclable construction materials and products under the Extended Producers Responsibility (EPR) policy that is enacted in the European Union for sustainable management of waste streams. Adhering to the EPR is difficult for buildings as they are long-term and complex assets. A high degree of transparency, accuracy and security is required to correctly track the lifecycle information of building parts and their respective manufacturers for the EPR implementation. For this purpose, a framework is conceptualised based on the immutability and transparency of blockchain technology to remove trust and trace barriers in the current supply chain. The proposed conceptual model results from the synergy of Building Information Modelling (BIM) technology, material and component banks, blockchain technology and smart contracts for the EoL treatment of recyclable materials. As a result, a datadriven and closed-loop material cycle will be accomplished. This paper demonstrates that through self-executing smart contracts, a clear line of responsibility and ownership could be defined while manufacturers could be made accountable in the postconsumer phase of their construction products.
\end{abstract}

Keywords: Extended Producer Responsibility; Circular Economy; Recycle; Resource Efficiency; Construction and Demolition Waste Management; Material Bank; Building Information Modelling; Blockchain; Smart Contracts

\section{INTRODUCTION}

In order to decarbonise the urban environment and meet the goals of the Paris Agreement by 2050, as well as in accordance with the Circular Economy Action Plan, the European Union (EU) has set rigorous goals to move towards radical resource efficiency and circular material flows. The construction industry is one of the largest industries with vast material flows, including raw and secondary materials, and Construction and Demolition Wastes (CDW). About $9 \%$ of the Gross Domestic Product (GDP) in the EU comes from the construction industry that uses almost $50 \%$ of all extracted materials as input. On the output side, CDW constitutes up to $30 \%$ of the EU waste stream, although EU member states have various CDW recycling and material recovery strategies [1][3]. Proper management of CDW requires sustainable and circular End-of-Lifecycle (EoL) treatment of construction materials. To this end, several environmental policies and metrics have been enacted, which emphasise on the role of the EoL handling scenarios on the overall environmental impacts of construction products.

The revised EN 15804 standard encourages the impacts of the EoL phase of construction materials and products, and the benefits of end-of-life recycling to be declared in the Environmental Product Declarations (EPDs). The EPD aims to communicate the environmental and human impacts of a product throughout its lifecycle. Moreover, the EPD makes the comparison of construction products easy as it is based on the Life Cycle Assessment (LCA) results [4], [5].

Furthermore, after the launch of the "Single Market for Green Products" initiative as a response to the "Resource Efficient Europe" strategy, the European Commission introduced the Product Environmental Footprint (PEF), which has similar EoL allocation considerations as the EPD. At the European level, the PEF aims to reduce the negative environmental impacts of construction products as long as they are in the supply chain. PEF is a voluntary product declaration, which means that producers have to come forth honestly with 
their knowledge of their products' composition and externalities [3]. There are significant efforts in the industry to harmonise PEFs and EPDs, as well as to ensure the quality and transparency of the LCA studies employed for these sustainability performance schemes [6].

The two previously described schemes demand the EoL phase's sustainable impacts to be considered along with other lifecycle phases. However, the Extended Producer Responsibility (EPR) policy directly targets the EoL management of the materials. The EPR is one of the pillars of the circular economy and aims to tackle the ever-increasing waste generation issue. Moreover, its principle is advocated by the Organisation for Economic Co-operation and Development (OECD). This principle makes manufacturers and importers of products responsible for the environmental impacts of their products throughout the whole lifecycle. According to the EPR, producers are also responsible for financial and/or physical treatment or disposal of post-consumer products in the EoL phase. This includes the collecting, sorting and recycling of materials [7]. The EPR was introduced in the 1980s to redefine waste handling responsibilities and to shift the burden of EoL treatment from the governmental level to the producer level. EPR can be achieved through regulatory, economic, or information instruments as well as take-back systems. The EPR encourages designers to consider the EoL scenarios in the inception of a project in order to prevent waste production at the source as well as to contribute to structured waste management at the end. EPR also promotes Design for Environment (DfE), which includes a range of environmental impact reduction strategies. The EPR policy is also known by other names, including "polluter pays principle (PPP)," "product stewardship," "product take-back," or "producers' pay" [8]. Many producers collaborate through Producer Responsibility Organisations (PROs), where they all pay the fees to a waste management company to handle collecting and recycling of products [7]. Producers seek to recover these costs by integrating them into the purchase price of the products. One can say that it is in fact the users of the products who pay for EoL handling in the end [8]. Currently, the EPR is actively fulfilled for the product categories of tyres, batteries, end-oflife vehicles, and waste of electrical and electronic equipment (WEEE or e-waste). However, buildings are i) extremely longterm assets and ii) extremely complicated assets with multiple owners and producers. Thus, it is challenging to execute and monitor the EPR policy in the building sector. However, through the use of digital technologies, we would like to offer a new conceptual model on how it could be possible to apply the EPR to the EoL of recyclable construction materials despite previous implementation hardships.

The above policies have been introduced to regulate the EoL phase and encourage resource efficiency in the current construction material market. However, a shortcoming is in the willingness, or even capability, of the current market to consider the EoL of the materials in an unknown future. Secure and tamper-proof lifecycle information and forward-looking EoL guidelines are necessary to fulfil the EPR and to make sure that the recycling responsibility will be supported by the policies and incentives, and acted upon by the manufacturer at the right time. Furthermore, having accurate data about the amount of CDW, recyclables, or reusable materials in the economy will create a regulated and reliable market. Previous studies delineated that knowing the exact type and amount of materials and scraps in the supply chain affects the scrap prices due to full transparency in the supply and demand, which could indirectly influence the recycling policies [9]. As a result, this will improve the environmental and economic indicators, which would then lead to accurate, forward-looking decisions for our society.

\section{RELATED WORK}

In this section, we introduce some important concepts necessary for our framework proposal. In the first subsection, we review Building Information Modelling (BIM), followed by Materials and Components Bank (M/C Bank), blockchain technology as well as smart contracts.

\section{A. Building Information Modelling (BIM)}

Building Information Modelling (BIM) is a methodology to create a 3D, digital representation of a building in which all of the building information is stored, managed and communicated. This digital model, also known as BIM or BIModel, is employed throughout the building lifecycle, from the pre-construction phase to the construction, maintenance, and deconstruction phases [10]. BIM offers many benefits, including the automation of repetitive tasks that would lead to fewer mistakes in the construction processes. The digitalisation of construction information through BIM creates a gateway for the construction industry to be linked with other digital technologies such as Artificial Intelligence (AI), Internet of Things (IoT), and blockchain. A BIModel acts as a data lake since it can accumulate the building information and deliver a structured data output to all stakeholders, which makes it a perfect link between the different technologies available. Although BIM manages and stores the data, it does not authenticate the data. However, data authenticity and integrity is possible through the synergy of BIM and blockchain technology. This synergy offers a secure way for data ownership and tracking [11]. A number of existing studies in the literature have examined the integration of BIM and blockchain networks, which led to several proposed models. This includes the studies from [12]-[14]. Their works and this paper make use of BIM to securely store information in a blockchain network.

\section{B. Materials and Components Bank (M/C Bank)}

The circular economy aims to close the material loop by keeping the materials in the economic value chain as long as possible without generating unnecessary waste. The concept of the M/C Bank was proposed by [15] to realise this zero-waste objective in the Architecture, Engineering, Construction, Owner and Operator (AECOO) industry. Rather than demolishing the building at the end of the lifecycle and landfilling the materials, the building can be deconstructed in such a way that building parts are further reused if they successfully pass the M/C Bank's structural, environmental and chemical performance assessment. In this case, the reusable materials should be prepared for reuse before their reintroduction to the supply chain [16]. Otherwise, they will be 
sent for recycling. M/C Bank is, in fact, a connection between the EoL phase and the new construction phase, as shown in Fig. 1. Deconstruction is the activity that is carried out in the EoL phase, which is followed by either Design for Deconstruction (DfD) for a new building or by design with reusable materials. A combination of these two design approaches is also possible [17]. While a physical M/C Bank can be anticipated for testing or storage purposes, the emphasis of this paper is on the digital M/C Bank, which stores all the lifecycle information of building components. The digital $\mathrm{M} / \mathrm{C}$ Bank is connected with BIModels to exchange the lifecycle information [18]; material passports and EPDs can also be linked to both BIM and M/C Bank.

\section{Blockchain Technology}

Blockchain technology uses a decentralised database contrary to the regular central databases. Blockchain technology is a particular case of the Distributed Ledger Technology (DLT), owing to the fact that it was born as a response to having secure digital financial transactions without a need for a central, trusted intermediary. Ledgers are trustable and systematic record-keeping tools in which not only financial entries but also ownership, status, authority and identity are registered [19]. The security of blockchain stems from three underlying cryptographic features: the blockchain protocol, private key encryption and the peer-to-peer network (i.e. P2P). These features ensure secure storage and transfer of any type of transaction on the nodes and within the blockchain network, respectively. A consensus mechanism is defined for the blockchain network, based on which a set of recorded transactions are validated, hashed and added to the chain after all the nodes (i.e. peers) accept the transactions. Thus, the transactions are distributed between nodes, and although users have similar rights within the blockchain, each would only see a part of the ledger. Fig. 2 illustrates how blocks of transactions are created, verified and added to a blockchain network. The consensus mechanism is an essential part of the blockchain technology as it eliminates the need for trustable intermediaries to validate the authenticity of the data.

Currently, blockchain technology is in constant development through fusion with different sectors, e.g., supply chain, logistics, health care. It is a relatively new technology and its future growth highly depends on its synergy with other technologies. Similarly, the potential of blockchain can be discovered through the integration of blockchain with other industries to create new conceptual models and use cases.

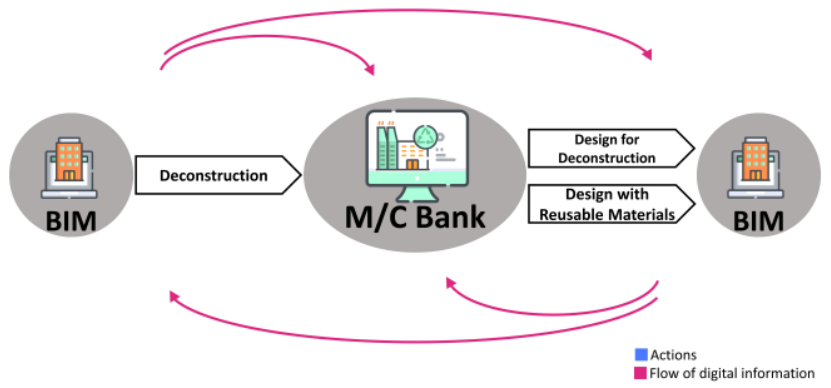

Fig. 1 The interaction of BIModels and the M/C Bank for circulation of materials' information during EoL and new design stages

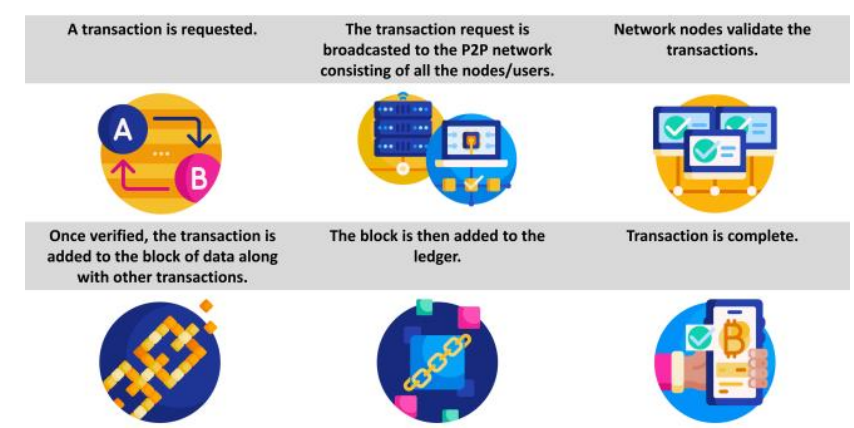

Fig. 2 The mechanism of blockchain

The implementation of blockchain technology in the construction sector has some limitations. For one, the industry is not tech-savvy, nor does it have proper digital foundations for smooth integration with blockchain. The construction industry is still in the transition to BIM, for which a great deal of time and money is spent by companies, either directed towards software purchases or training.

\section{Smart Contracts}

The focus of blockchain technology shifted from financial value transactions to other types of value as it progressed over time. In a business case, there may be other vital conditions aside from the monetary value when executing a transaction. For instance, a transaction may involve specific rules, policies, laws, and regulations. For allowing these other real-world constraints to be realised on a blockchain, smart contracts add a layer of computation logic to the network. Therefore, through the smart contract, a wide variety of decentralised applications and innovative features can be implemented on the blockchain.

Smart contracts are a set of coded protocols registered in a blockchain that is automatically executed by the computer once the contract triggers are set off. An example of a smart contract is shown in Fig. 3. They self-execute the actions that are anticipated for conditions, which verified parties have agreed upon. Similar to other transactions in a blockchain network, once the smart contract is executed, it is deployed and invoked on the network before it is validated by users through a consensus process. The elimination of human bias in contract employment increases the trust and fairness between participants.

\section{FRAMEWORK IMPLEMENTATION}

One of the common aims of the EU-wide directives and initiatives is to divert CDW and valuable recyclable materials from landfills, to turn waste into resources and to reduce raw material extraction [20], [21]. Recycling is one of the crucial cycles in materials' lifecycle in order to revalorise the materials and to re-inject them back into the economy. One of the

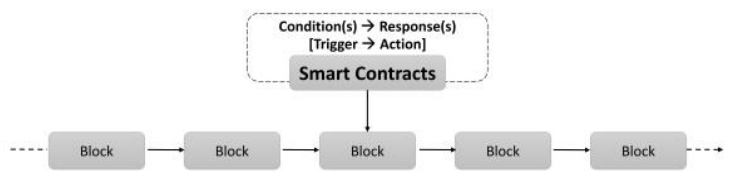

Fig. 3 The mechanism of Smart Contracts in a blockchain network 
policies that directly addresses this issue is EPR. However, the EPR policy is not followed up in the post-consumer phase of buildings, i.e. the deconstruction phase, due to difficulties regarding the lifecycle and EoL information tracking of the plethora of materials that exist in every single building. This has caused many valuable materials to end up in landfills or to be poorly recycled. Furthermore, the lack of clear information about the whereabouts of the discarded materials has resulted in a grey area in terms of the unknown physical volume as well as the financial value of the existing CDW. Thus, the objective of the proposed framework here is to revalorise the recyclable construction materials and to define clear lines of responsibility regarding the waste management and EoL handling throughout the lifecycle of a construction product with a particular focus on EoL information. To this end, a smart EoL handling system with a take-back mechanism is conceptualised in this paper to tackle the EoL problems. This conceptual model makes the EPR implementation and monitoring within the building sector possible by using blockchain technology and smart contracts in connection with both M/C Bank and BIM technology.

The expected service life of a building can go up to 50 years. In many cases, buildings are in the operation phase long after the designated service life. Information loss, decay and tampering as well as digital obsolescence threaten the integrity and authenticity of the lifecycle information due to this lengthy time perspective. It is imperative for any long-term lifecycle information management strategy to consider a secure database to protect the data against the above threats. In addition, to encourage construction material manufacturers to take up the EoL responsibility of their products, they need a trustable information loop regarding the EoL data of their products. A secure way to keep the lifecycle information over the long time frame described is to utilise the capabilities of blockchain to track items throughout the supply chain. Hence, the conceptual model in this paper benefits from smart contracts that run on the blockchain network to automatically execute a set of predefined actions when the building is decommissioned.

So far, EPR and material construction take-back systems have not been implemented in the building sector for several reasons. Firstly, it is not easy nor is it common practice for construction material manufacturers to keep consistent and future-proof records of the whereabouts of their sold inventory for about a century, if not more. It is not easy for producers to keep in touch with all their previous clients in order to fulfil their extended producer responsibilities. Secondly, contrary to other EPR categories, e.g., vehicles or batteries, a construction material producer usually sells the materials to a construction contractor, not the final building owner/user. Ownership issues add a layer of complexity to a take-back system, especially if EPR-based financial deposit-refund instruments are also in place. While the initial contractor might not be in the deconstruction scene in a future scenario, the third issue is related to producers who are no longer active. If they are not in business anymore, how can they fulfil their EoL responsibility, or who should shoulder this burden instead of them? A highlighted issue in the EPR policy is the existence of "orphan products." These are products that are left orphaned as no party takes the responsibility for their EoL handling, or other producers have to take care of them [7]. These complexities can be overcome by systematically using smart contracts and updating ownerships, EoL responsibilities or claims. A smart contract-based system can organise an accountability system where producers and contractors can settle on their EoL responsibility share before exiting the business value chain and leaving orphan goods behind. This will increase the market's positive competitiveness as a result of minimising the free-rider problem, where the burden of recycling is not only on the ecologically responsible companies. Fig. 4 illustrates the mechanism of the proposed conceptual framework. It demonstrates different smart contract triggers and follow-up actions.

The EoL phase begins when the product is disassembled from the building until the last step of its proper treatment. Hence, both the M/C Bank and the producers will handle the EoL treatment responsibility. Initially, when a building is deconstructed, materials and components are sent to the $\mathrm{M} / \mathrm{C}$ Bank for reusability assessment. Upon arrival of the materials at the $\mathrm{M} / \mathrm{C}$ Bank, a smart contract is automatically executed to notify the original material/product producer that their product is no longer nested in a building but in the custody of the $\mathrm{M} / \mathrm{C}$ Bank for assessment. Based on the assessment report, the materials are either suitable for further reuse or recycling. If the product is reusable, a smart contract notifies the producer that their product is still in the supply chain and will be used further (path number 1 in Fig. 4). This entails a series of updates in the smart contracts to prepare them for a future take-back scenario in the next lifecycle of products. Otherwise, if the material is to be recycled, another clause of a smart contract will be triggered, which notifies the producer about this judgement as well as their duty regarding the EoL treatment of their products. However, as mentioned earlier, the manufacturer might not be in the business by the time the building is being

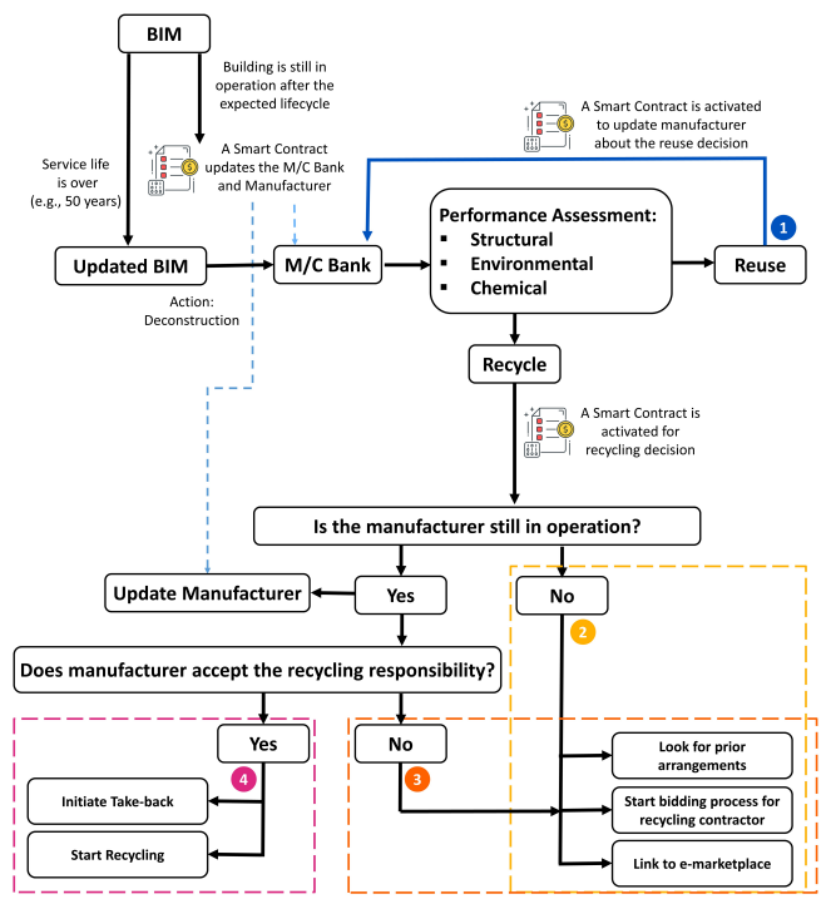

Fig. 4 Proposed conceptual framework for end-of-life recycling of construction materials 
deconstructed. Thus, a smart contract clause is activated to enquire whether the producer is in operation after the service life is over and if they are able to fulfil their extended responsibilities (path number 2 in Fig. 4). Nevertheless, even if producers are still operational, they are free to choose if they will accept the EoL responsibility (path number 3 in Fig. 4). If not, they should make arrangements with the M/C Banks, other producers, or PROs to update the smart contracts before the time of the deconstruction. Also, the M/C Bank can start a bidding process to find a suitable recycling contractor. These provisions ensure that the framework is inclusive and has few entry barriers. Even if the producers have no means to fulfil their extended EoL responsibility personally, they can contribute to the system by making prior arrangements and/or sharing the important EoL guidelines regarding their products. In such cases, an integration of this conceptual model with emarketplaces is also feasible. Blockchain-based e-marketplaces are new research hotspots [22]. There could be cases where a material or component can be upcycled and reused in another context without significant performance modifications. Therefore, this is an excellent opportunity for cross-industry collaboration for creating new inner cycles in the material loop whilst generating new use cases and business revenues. In fact, the new European Green Deal encourages cooperation across value chains for having a strong and integrated single market for secondary raw materials and by-products [23].

In the ideal case where the producers are active and willing to fulfil their responsibilities, the next smart contract clause will be triggered to initiate the take-back mechanism and deliver the recyclables back to the producers (path number 4 in Fig. 4). They will recycle the materials and inject them back to their business processes. In this mechanism, it is the responsibility of the $\mathrm{M} / \mathrm{C}$ Bank to administer the EoL treatment. At the same time, the producers are responsible for the financial and physical treatment of the materials, as stated in the EPR policy.

Blockchain network provides an infrastructure for a smart, non-discriminative, inclusive, trustable and agile EoL treatment ecosystem, where the biases and errors are minimised since smart contracts administrate the decisions and notifications. Producers know that they can trust such a system for on time and correct information. As everything is recorded in smart contracts, producers are relieved from the burden of tracking their products or verifying the lifecycle information and can come to the EoL scene only when the materials are assessed to be recycled. On top of that, as ownerships are already handled through smart contracts, it would be easy for producers to redeem their products without additional administrative or legal issues. Saving all stakeholders from unnecessary paperwork and claims will create efficiency in the whole recycling and EoL treatment system.

In an ideal situation, as manufacturers know the exact location and status of the materials, they can collaborate with the $\mathrm{M} / \mathrm{C}$ Bank for taking back and recycling the materials, hence, fulfilling the EPR in the construction sector. However, to avoid confusions and to regulate the interaction of different parties, further smart contract clauses can be anticipated to i) prevent the producers from claiming their product before the EoL phase, as well as to ii) prevent users/clients from treating materials carelessly since they are not responsible for the EoL treatment. For example, at the end of the expected service life of a building, a smart contract automatically sends messages to the M/C Bank to enquire the status of the building; whether it will be deconstructed or the owners will use it beyond the expected lifecycle. In the latter case, the M/C Bank will estimate a new expected service life and the smart contract will be updated accordingly. Also, in case of an accident, e.g. fire, a smart contract will automatically report the damages to insurance companies and will update the manufacturers about the incident. Following that, if the damages make dwelling impossible in that particular building, it can be deconstructed before the end of the expected service life. Thus, manufacturers will receive automatic notifications to take back the recyclables earlier than expected.

Finally, Fig. 5 shows a possible way on how BIM fits in this conceptual framework and how it connects different stakeholders. BIM is the only way to date to digitise the building components in an integrated way that can later be linked with other technologies. All of the lifecycle information is intermittently updated and stored in a BIModel, which is then linked to the M/C Bank. Thus, the M/C Bank must be a partner in the smart contracts, while other partners in these should be the producers and consumers (building contractors and users). This is beyond the scope of this paper to go over the complex issue of who really should be considered as the consumer, building contractors or end-users. Nevertheless, there could be other possible ways to integrate BIModels and smart contracts which will need to be further investigated.

The proposed conceptual model offers quite a few benefits due to the synergy of the involved digital technologies. Essential lifecycle information that is required for the $\mathrm{M} / \mathrm{C}$ Bank's assessment is securely kept, no more information loss or decay. Due to all the technologies involved, the paperwork involved is considered as low, especially when it comes to tracking the EoL responsibility and implementing EPR in the construction sector. This leads to the next benefit, which is the automatic on-time messaging. Every party in the supply chain will be notified about their EoL responsibilities and the status of their products. As a consequence of this automation, the need for a central authority to administer the take-back notification is minimised. Also, there is no bias in informing the right producer at the right time and deliver them the right materials. Human errors are, thus, minimised, and the efficiency of the whole EoL take-back system is increased. The

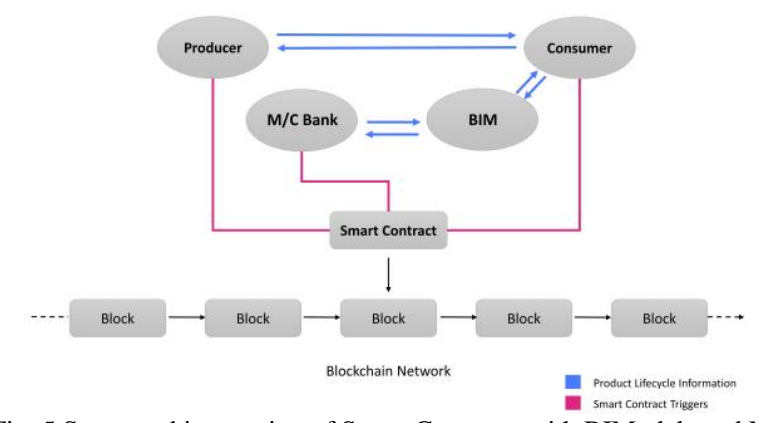

Fig. 5 Suggested integration of Smart Contracts with BIModels and M/C Bank to update producers and consumers about the status of materials 
automatic tracking of lifecycle information and EoL handling responsibilities eradicates the orphan goods issue that is common in all industries.

With enough clarity about the material input and output, the statistics about the CDW, recyclables, as well as reusable materials will be more accurate. This accuracy would benefit environmental metrics and indices, such as Net Additions to Stock (NAS) [24]. NAS suffers from a lack of data about the gross additions and removals from the physical stock of a community, which are input and output building materials in building stock.

The suggested framework is adaptive. It can be applied to other industries that also suffer from a lack of transparency and statistics in their supply chain or to those that need to regulate the EoL of their products.

Realising a digital circular economy needs to start from conceptual models where a combination of technologies are directed to solve a circular issue. On that note, this framework aims to use digital technologies to create a robust and trustable take-back system for construction materials in order to recover the value of recyclable materials. A financial blockchain-based mechanism will be introduced in the next phase of this study to create a win-win solution for the producers and consumers. This solution adheres to the EPR financial instruments and encourages more construction stakeholders to take back their products at the EoL phase for recycling.

\section{CONCLUSION}

The present paper offers a digital circular economy solution for the supply chain of recyclable materials in the construction industry. The proposed conceptual model centres on a product take-back system by defining clear lines of responsibility in the EoL phase of buildings, which is also in line with the EPR policies.

In the EoL phase of facilities and after the deconstruction, materials are sent to the $\mathrm{M} / \mathrm{C}$ Bank for performance assessment. Whether suitable for further reuse in a second lifecycle or suitable for recycling, the producers of the materials will be automatically notified by smart contracts about this step and the assessment outcome. For recyclable materials, further smart contract clauses will be activated that enable producers to take back their materials or components. Through this smart-contact-based system, producers will be accountable for the EoL handling of their products. This mechanism would enable the implementation of the EPR policy in the construction sector, which is not the case at the moment. Hence, "construction" producers would be made responsible for the treatment of their products and materials at the end of their lifecycle.

One reason why EPR has not been implemented in the construction sector is the long service life of buildings, as they can be used for, e.g., 50 years or more. Moreover, the complexity and the multitude of materials in a building makes EPR implementation improbable due to the extreme amount of paperwork for tracking lifecycle status and ownership responsibilities. By using a combination of digital technologies, including BIM and blockchain, we believe that we can overcome the above challenges.

Knowing the exact type and amount of materials in the supply chain, either raw or secondary, will affect scrap prices because of full transparency in supply and demand. This transparency will benefit the environmental metrics to draw an accurate picture of the existing $\mathrm{CDW}$ or materials in the market.

Furthermore, by using blockchain networks, the information will be immutably and securely stored and protected against digital attacks or decays. Consequently, human errors will be reduced, and the efficiency and accuracy of the system will be increased. The novelty of this framework is in the deployment of smart contracts and decentralised blockchain network to ensure the long-term security of data as well as to make it easy to create financial incentives on top of take-back mechanism for producers, manufacturers and consumers. Having a monetary incentive attracts more AECOO stakeholders to participate in planning for a future EoL. With blockchain, it is possible to create financial value for the materials in the EoL phase for the mutual benefit of all society members, while increasing the sustainability and circularity of the construction sector.

A future direction for this framework is to investigate what type of blockchain network would be suitable for such a use case. Another direction to follow in future is to work on the practical integration of BIM and Smart Contracts.

Overall, in this paper, we showed how blockchain technology can be used in synergy with BIM technology to create secure and automatic lifecycle information exchanges between AECOO actors who join the M/C Bank schema. Subsequently, the proposed model paves the way for monitoring and fulfilling the extended responsibility of construction material producers as outlined by the EPR policy.

\section{ACKNOWLEDGMENT}

This research was carried out as part of the EcoConstruction for Sustainable Development (ECON4SD) project that is supported by the Investissement pour la Croissance et l'emploi-European Regional Development Fund (2014-2020), with the grant agreement 2017-02-015-15.

\section{REFERENCES}

[1] European Commission. The European construction sector - A global partner, European Commission, Brussels, Ref. Ares (2016) 1253962 11/03/2016, 2016.

[2] European Commission. Construction and demolition waste (CDW), http://ec.europa.eu/environment/waste/construction_demolition.htm (Access date: 02/05/2019).

[3] European Commission. Communication from the Commission to the European Parliament, the Council, the European Economic and Social Committee and the Committee of the Regions- Roadmap to a Resource Efficient Europe, European Commission, Brussels, COM (2011) 571 final, 2011.

[4] CEN/TC350. Sustainability of construction works - Environmental product declarations - Core rules for the product category of construction products, European Committee for Standardization, EN 15804:2012+A2:2019, 2019. 
[5] ISO, Environmental labels and declarations - Type III environmental declarations - Principles and procedures, International Organization for Standardization, ISO 14025:2006, 2006.

[6] V. Durão, J. D. Silvestre, R. Mateus, and J. de Brito, "Assessment and communication of the environmental performance of construction products in Europe: Comparison between PEF and EN 15804 compliant EPD schemes," Resour. Conserv. Recycl., vol. 156, pp. 104703, May 2020, doi: 10.1016/j.resconrec.2020.104703.

[7] OECD, Extended Producer Responsibility: Updated Guidance for Efficient Waste Management. Organisation for Economic Co-operation and Development, 2016.

[8] K. H. Forslind, "Implementing extended producer responsibility: the case of Sweden's car scrapping scheme", J. Clean. Prod., vol. 13, no. 6, pp. 619-629, May 2005, doi: 10.1016/j.jclepro.2003.12.017.

[9] D. T. Kaffine, "Scrap Prices, Waste, and Recycling Policy," Land Econ., vol. 90, no. 1, pp. 169-180, Feb. 2014, doi: 10.3368/le.90.1.169.

[10] R. Sacks, C. Eastman, G. Lee, and P. Teicholz, BIM handbook : a guide to building information modeling for owners, managers, designers, engineers and contractors, 3rd ed. Hoboken, New Jersey: Wiley, 2018.

[11] Ž. Turk and R. Klinc, "Potentials of Blockchain Technology for Construction Management," Creat. Constr. Conf. 2017 CCC 2017 19-22 June 2017 Primosten Croat., vol. 196, pp. 638-645, Jan. 2017, doi: 10.1016/j.proeng.2017.08.052.

[12] A. Fitriawijaya, T. Hsin-Hsuan, and J. Taysheng, A blockchain approach to supply chain management in a BIM-enabled environment, in Intelligent and Informed - Proceedings of the 24th International Conference on Computer-Aided Architectural Design Research in Asia, CAADRIA 2019, New Zealand, 2019, vol. 2, pp. 411-420.

[13] A. Shojaei, I. Flood, H. I. Moud, M. Hatami, and X. Zhang, "An Implementation of Smart Contracts by Integrating BIM and Blockchain", in Proceedings of the Future Technologies Conference (FTC) 2019, Cham, 2020, pp. 519-527, doi: 10.1007/978-3-030-325237_36.

[14] A. Hijazi, S. Perera, A. Alashwal, and R. Calheiros, "Enabling a Single Source of Truth Through BIM and Blockchain Integration," in Proceedings of the 2019 International Conference on Innovation, Technology, Enterprise and Entrepreneurship (ICITEE 2019), Bahrain, 2019, pp. 385-393.

[15] G. Cai and D. Waldmann, "A material and component bank to facilitate material recycling and component reuse for a sustainable construction: concept and preliminary study," Clean Technol. Environ. Policy, vol. 21, pp. 2015-2032, Nov. 2019, doi: 10.1007/s10098-019-01758-1.

[16] European Commission. EU Construction \& Demolition Waste Management Protocol, European Commission Directorate-General for Internal market, Industry, Entrepreneurship and SMEs, Brussels, 2016.

[17] A. Akbarieh, L. B. Jayasinghe, D. Waldmann, and F. N. Teferle, "BIMBased End-of-Lifecycle Decision Making and Digital Deconstruction Literature Review," Sustainability, vol. 12, no. 7, Art. no. 7, Jan. 2020 doi: 10.3390/su12072670.

[18] L. B. Jayasinghe and D. Waldmann, "Development of a BIM-Based Web Tool as a Material and Component Bank for a Sustainable Construction Industry," Sustainability, vol. 12, no. 5, Art. no. 5, Jan. 2020, doi: 10.3390/su12051766.

[19] D. Tapscott and A. Tapscott, Blockchain revolution: how the technology behind bitcoin is changing money, business, and the world. New York: Portfolio, Penguin, 2016.

[20] European Parliament and Council. Directive 2008/98/EC of the European Parliament and of the Council of 19 November 2008 on waste and repealing certain Directives, Brussels, L 312/3, 2008.

[21] European Commission. Report from the Commission to the European Parliament, the Council, the European Economic and Social Committee and the Committee of the Regions - on the implementation of the Circular Economy Action Plan, European Commission, Brussels, COM (2019) 190 final, 2019.

[22] S. Lawrenz, P. Sharma, and A. Rausch, "Blockchain Technology as an Approach for Data Marketplaces", in Proceedings of the 2019 International Conference on Blockchain Technology, New York, NY, USA, 2019, pp. 55-59, doi: 10.1145/3320154.3320165.
[23] European Commission. Communication from the Commission to the European Parliament, the European Council, the Council, the European Economic and Social Committee and the Committee of the Regions the European Green Deal, European Commission, Brussels, COM (2019) 640 final, 2019.

[24] Eurostat. Economy-wide material flow accounts handbook, Luxembourg: Publications Office of the European Union, 2018. 